\title{
Credibility Judgment Predictors for Child Sexual Abuse Reports in Forensic Psychiatric Evaluations
}

\author{
Ling-Hsiang Wang', Yu-Yung Hung1, Philip C. Chow², Che-Sheng Chu', \\ Hsing-Jung $\mathrm{Li}^{3}, \mathrm{Ti}^{\mathrm{Lu}}{ }^{1}$, and Ching-Hong Tsai ${ }^{3,4} \bowtie$ \\ ${ }^{1}$ Department of Psychiatry, Kaohsiung Veterans General Hospital, Kaohsiung, Taiwan \\ ${ }^{2}$ Department of Psychiatry, Conde de São Januário Central Hospital, Macau, China \\ ${ }^{3}$ Child \& Adolescent Psychiatry Department, Kaohsiung Municipal Kai-Syuan Psychiatric Hospital, Kaohsiung, Taiwan \\ ${ }^{4}$ Graduate School of Human Sexuality, Shu-Te University, Kaohsiung, Taiwan
}

Objective We intended to analyze the credibility judgment in written forensic psychiatric reports of child sexual abuse registered in Southern Taiwan.

Methods Ninety-six cases of child sexual abuse between August 2010 and October 2017 encountered in two hospitals were analyzed. The results in these reports were categorized into credible and non-credible. We identified the factors that distinguished between the two groups in bivariate analyses using chi-square test. A binary logistic regression analysis was performed to determine whether the factors that significantly correlated in the bivariate analyses were independent predictors of credible judgments.

Results Among 96 cases, 70 (73\%) were judged as credible. Consistent testimonies of children (odds ratio=40.82) and multiple abuse events (odds ratio $=6.05$ ) were positive variables independently related to the sexual abuse allegations judged as credible.

Conclusion The number of allegations judged as credible in this study was slightly higher than that reported in other studies. Our findings about predictors for credible cases are not in line with those reported previously. Due to the differences in resources of the cases and backgrounds of the evaluators among multiple studies, direct comparisons with previous studies must be treated with caution.

Psychiatry Investig 2019;16(2):139-144

Key Words Child sexual abuse, Expert testimony, Child psychiatry, Forensic psychiatry.

\section{INTRODUCTION}

In the majority of child sexual abuse (CSA) cases, a lack of definite medical or physical evidence means that children's testimony forms the crucial component of subsequent investigations. ${ }^{1,2}$ However, the limitations in cognitive function and language development of children make the assessment of witness credibility one of the most important and challenging aspects of forensic evaluation. ${ }^{3}$ The use of a structured protocol in interviews with CSA victims may facilitate child inves-

Received: June 20, 2018 Revised: October 10, 2018

Accepted: December 3, 2018

$\triangle$ Correspondence: Ching-Hong Tsai, MD, PhD

Child \& Adolescent Psychiatry Department, Kaohsiung Municipal Kai-Syuan Psychiatric Hospital, 130 Kai-Syuan 2nd Road, Ling-Ya District, Kaohsiung City 80276, Taiwan, ROC

Tel: +886-7-751-3171, Fax: +886-7-716-1843

E-mail: chinghongtsai@gmail.com

(a) This is an Open Access article distributed under the terms of the Creative Commons Attribution Non-Commercial License (https://creativecommons.org/licenses/bync/4.0) which permits unrestricted non-commercial use, distribution, and reproduction in any medium, provided the original work is properly cited. tigators in making a judgment of credibility. ${ }^{4}$ However, some prior studies have shown that about a third of credibility judgments made on this basis tend to be incorrect. ${ }^{5,6}$ Further, although some instruments for assessing credibility have been developed (e.g., criteria-based content analysis), these have not yet been empirically validated. ${ }^{7}$ On the whole, no clear guidelines have been established for determining the credibility of CSA reported by children.

Some studies have explored the characteristics of the child and features of the abusive event, and how those are subsequently related to credibility judgment. For victims, being of older age and without cognitive delay are often predictive of credible testimony as judged by an investigator and the substantiation of abuse allegations. ${ }^{8-10}$ This is because children of younger age or those with cognitive delay may not have the verbal or cognitive skills to recognize the purpose of the forensic interview or to describe the abusive event during the interview in detail. ${ }^{11}$ Moreover, testimony from girls was more likely to be viewed as credible than that of boys, ${ }^{9,12}$ while a re- 
view study has proposed that this gender effect is minimized when the suspect is not the biological parent of the child. ${ }^{13}$ The family background of the victim also plays an important role in credibility assessment. One recent study has shown that it is more likely for the testimony given by the children of married parents to be judged as credible. ${ }^{8}$ Similarly, another study showed that abuse allegations involving custody or visitation disputes were less likely to be substantiated. ${ }^{10}$ Regarding the features of an abusive event, a single abuse event is predictive of credible testimony in children. ${ }^{8,14}$ Some studies have also attempted to examine the impact of abuse severity on witness credibility but revealed mixed results. ${ }^{8-10}$ It is likely that researchers adopt a variety of definitions when assessing the severity of abuse, and this could contribute to the inconsistent pattern of results. Considering the victim-perpetrator relationship, some researchers have argued that the testimony of victims abused by strangers might be judged more credible than that of victims abused by someone already known to them; ${ }^{15}$ however this can be vice-versa in some cases. ${ }^{16}$

The American Academy of Child and Adolescent Psychiatry has developed practice parameters to assist forensic evaluation conducted during CSA allegations. ${ }^{17}$ However, most recent research on credibility assessment and related factors has been conducted by out-of-hospital mental health or legal professionals. Thus far, there has been relatively little research in the area from the psychiatric point-of-view. In Taiwan, forensic psychiatric evaluation is already a part of hospital psychiatry services. As an expert witness, the child psychiatrist must often help the prosecutor assess the credibility of CSA allegations and present the deduction in a written forensic psychiatric report. Hence, the purpose of this study was to examine the child characteristics and abuse event-related features that might predict child credibility in the written forensic psychiatric report. We hypothesized that there would be discrepancies between the perceptions of child psychiatrists compared with other professionals regarding credibility judgment.

\section{METHODS}

\section{Data source and study sample}

The integrated program for the forensic investigation of CSA allegations and psychiatric evaluation of victims has been adopted in Kaohsiung City since 2010. ${ }^{18}$ The multidisciplinary team (MDT) of the psychiatry department can help a district prosecutor with the initial forensic interview and the subsequent credibility evaluation. The MDT includes a child psychiatrist, a clinical psychologist, and a social worker. In the first part of the MDT approach program (Figure 1), the district prosecutor interviews the child victim to acquire a testimony relating to the possibly abusive event. The struc-

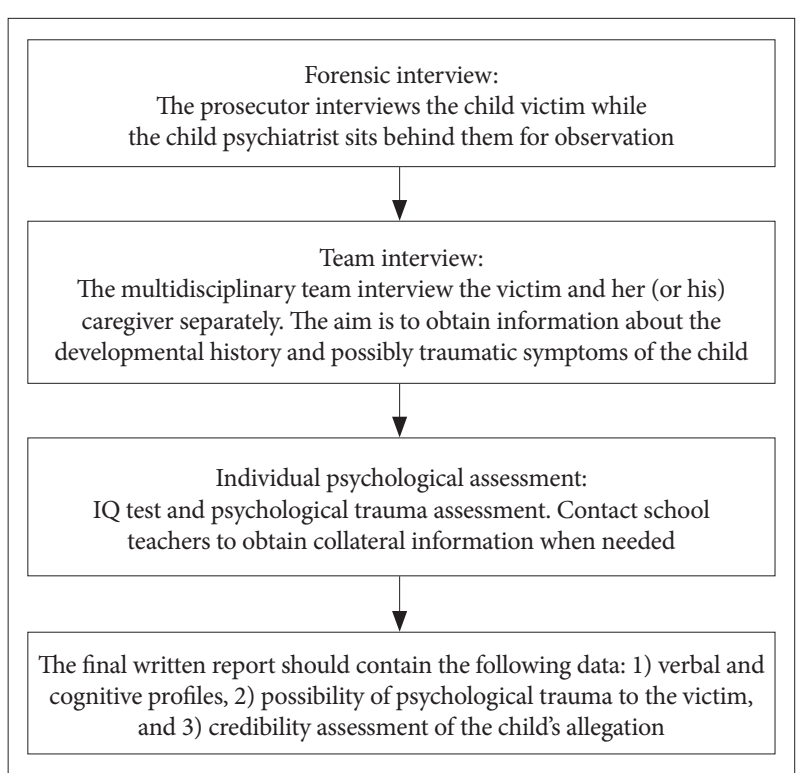

Figure 1. Procedure of multidisciplinary team approach.

tured interview protocol has been translated into Mandarin and introduced widely throughout Taiwan, ${ }^{19}$ and increasing numbers of legal professionals are required to undertake the training course and practice it. During the forensic interview conducted by the prosecutor, the child psychiatrist sits behind to observe and gives advice to facilitate the process as needed. Upon completion of the evaluation, the child psychiatrist must analyze the credibility of the CSA allegations in the written forensic psychiatric report. Due to the lack of standardized assessment tools, the child psychiatrist decides credibility based on their clinical and professional judgment.

In this study, a retrospective review was conducted of the records of sexually abused children who were referred to two designated hospitals by the Kaohsiung District Prosecutors Office between August 1, 2010, and October 31, 2017. Data were obtained from the written forensic psychiatric reports for each child. The Institutional Review Board of Kaohsiung Municipal Kai-Syuan Psychiatric Hospital approved the research (KSPH-2017-29). Since this was a retrospective study and personally identifiable information was removed from the reports before we received them, informed consent from victims or their caregivers was not required. Cases of victims aged $>18$ years or who lacked a child psychiatrist's assessment on credibility in the final report were excluded. Ninetysix cases ( $89.6 \%$ females) in the age range of $2-16$ years ( $7.03 \pm 3.53$ years) were included. Most child psychiatrists defined a credible testimony as an honest and grossly correct statement made by the victim in the forensic interview. Credibility assessments in these reports were classified by the research team into one of the two categories: 1) credible and 2) non-credible (meaning incredible or indeterminable). We then 
compared the data obtained from these two groups.

\section{Data collection}

\section{Demographic data}

Demographic data, including age at first interview, sex, education, the family structure at first interview, and assessment site, were gathered.

\section{Psychopathology}

A board-certified child and adolescent psychiatrist conducted a diagnostic interview based on the Diagnostic and Statistical Manual of Mental Disorders, 4th Edition, Text Revision (DSM-IV-TR). ${ }^{20}$ IQ was measured by a clinical psychologist using the Wechsler Intelligence Scale for Children, 4th Edition, or the Wechsler Preschool and Primary Scale of Intelligence, Revised, as appropriate. ${ }^{21,22}$ The team examined for psychiatric disorder and/or intellectual disability in each child.

\section{Features of an abusive event}

Features of the examined abusive events were as follows: the approximate duration to delayed disclosure (time from abuse onset to the first interview), number of abuse events (once or multiple), involvement of vaginal or anal penetration, perpetrator-victim relationship, familiarity of the victim with the perpetrator (the child could recognize the perpetrator's identity without even knowing the perpetrator's name), and whether the perpetrator threatened the victim with serious consequences as a result of disclosure.

\section{Report consistency}

We observed that the consistency of the statement was usually discussed in the final written report. Corwin defined the indicator as "Consistency in reporting major facts of sexual victimization, minor details may vary."23 Most of the child psychiatrists adopted this or a similar approach, and assessed the consistency of statements across or within interviews during the entire program. Thus, we coded this variable based on the decision in each report.

\section{Statistical analysis}

Testimonies were classified into two groups: credible vs. noncredible. Between-group comparisons for categorical variables were analyzed using either chi-square test or Fisher's exact test. Next, a binary logistic regression analysis was conducted to examine whether the significant variables in the bivariate analyses were independent predictors of credible testimony. As the age of the victim has been shown to be a fundamental factor related to perceived credibility, ${ }^{8,9,11,13}$ we used this variable in the logistic regression equations regardless of whether or not the result was significant in the initial analysis. The level of statistical significance was set as two-tailed $p<0.05$. All analyses were conducted using SPSS software (IBM SPSS Statistics, 18th ed., SPSS Inc., Chicago, IL, USA).

\section{RESULTS}

Of the total cases, $72.9 \%(\mathrm{n}=70)$ were viewed as credible; $15.6 \%(\mathrm{n}=15)$, incredible; and $11.5 \%(\mathrm{n}=11)$, indeterminable. The average full-scale IQ of children was $82.33 \pm 22.90$. Corroborating evidence (e.g., medical evidence or other eyewitnesses) could be found in $27 \%$ of the cases but all cases were referred to our program for further evaluation.

\section{Bivariate relationships between variables and credibility}

Table 1 shows the epidemiologic and event characteristics of both groups and their relationships to perceived credibility. The testimonies of children who were cared for by both parents were viewed as less credible $(\mathrm{p}=0.006)$. Children's statements were deemed credible if they were familiar with the suspect ( $\mathrm{p}=0.017$ ), but the result could not be duplicated in cases where the abuser was a parent/parent figure. Regarding the event characteristics, there was a higher probability for a report to be evaluated as credible when the victim reported having experienced multiple abuse events as opposed to a single abuse event $(\mathrm{p}<0.001)$. Further, a perpetrator threatening the victim with serious consequences in case of any disclosure was significantly associated with credible statements $(\mathrm{p}=0.032)$. Finally, children's consistency in statements was highly associated with perceived credibility $(\mathrm{p}<0.001)$.

\section{Model for the prediction of credibility}

We used multivariate logistic regression to further examine the significant variables identified in the binary analyses. As stated above, age at first interview was an important variable to be used in the regression equation, regardless of the results obtained in the first analysis. The outcome of the logistic regression analysis in identifying predictors affecting perceived credibility is presented in Table 2 . The model was significant $(\mathrm{p}<0.001)$ and correctly classified $86 \%$ of the cases, with Nagelkerke's $R^{2}=0.644$. The consistency in the reports of children was the most powerful predictor of perceived credibility $(\mathrm{OR}=40.82, \mathrm{p}<0.001)$. In addition, the statements of children with multiple abuse events were deemed more credible than those reporting a single abuse event $(\mathrm{OR}=6.05, \mathrm{p}=0.046)$.

\section{DISCUSSION}

To our knowledge, this is the first study exploring the per- 
spectives of child psychiatrists on credibility judgments in CSA allegations. Instead of interviewing the child psychiatrists or conducting questionnaire-based research, the present study analyzed written forensic reports to identify the factors that impact upon psychiatrist judgment. Owing to the observation that only $23 \%$ of written credibility evaluations

Table 1. Descriptive statistics of current sample based on credibility

\begin{tabular}{|c|c|c|c|}
\hline Characteristics & $\begin{array}{c}\text { Credible } \\
(\mathrm{N}=70)\end{array}$ & $\begin{array}{c}\text { Non-credible } \\
(\mathrm{N}=26)\end{array}$ & $\mathrm{p}$ \\
\hline Assessment site (\%) & & & 0.503 \\
\hline General hospital & $21(30)$ & $6(23.1)$ & \\
\hline Psychiatric hospital & $49(70)$ & $20(76.9)$ & \\
\hline Child's age at first interview (\%) & & & 0.118 \\
\hline$<7$ years & $36(51.4)$ & $18(69.2)$ & \\
\hline$\geq 7$ years & $34(48.6)$ & $8(30.8)$ & \\
\hline Delayed disclosure (\%)* & & & 0.292 \\
\hline Within 1 year & $46(65.7)$ & $20(76.9)$ & \\
\hline$>1$ year & $24(34.3)$ & $6(23.1)$ & \\
\hline $\operatorname{Sex}(\%)$ & & & 1.000 \\
\hline Male & $7(10)$ & $3(11.5)$ & \\
\hline Female & $63(90)$ & $23(88.5)$ & \\
\hline Psychiatric diagnosis (\%) & & & 0.871 \\
\hline Yes & $23(32.9)$ & $9(34.6)$ & \\
\hline No & $47(67.1)$ & $17(65.4)$ & \\
\hline Mental retardation (\%) & & & 0.126 \\
\hline Yes & $16(22.9)$ & $10(38.5)$ & \\
\hline No & $54(77.1)$ & $16(61.5)$ & \\
\hline Caregiver (\%) & & & 0.006 \\
\hline Both parents & $18(25.7)$ & $14(56)$ & \\
\hline Others & $52(74.3)$ & $11(44)$ & \\
\hline Relationship with suspect (\%) & & & 0.931 \\
\hline Parent or parental figure & $33(47.1)$ & $12(46.2)$ & \\
\hline Non-parental figure & $37(52.9)$ & $14(53.8)$ & \\
\hline Familiarity with the suspect (\%) & & & 0.017 \\
\hline Yes & $57(81.4)$ & $15(57.7)$ & \\
\hline No & $13(18.6)$ & $11(42.3)$ & \\
\hline Number of abuse events (\%) & & & $<0.001$ \\
\hline More than one & $60(85.7)$ & $12(46.2)$ & \\
\hline One & $10(14.3)$ & $14(53.8)$ & \\
\hline Vaginal or anal penetration (\%) & & & 0.179 \\
\hline Yes & $35(50)$ & $9(34.6)$ & \\
\hline No & $35(50)$ & $17(65.4)$ & \\
\hline Threatened by the Suspect $(\%)^{\dagger}$ & & & 0.032 \\
\hline Yes & $11(15.7)$ & $0(0)$ & \\
\hline No & $59(84.3)$ & $26(100)$ & \\
\hline Consistency in report (\%) & & & $<0.001$ \\
\hline Yes & $57(81.4)$ & $2(7.7)$ & \\
\hline No & $13(18.6)$ & $24(92.3)$ & \\
\hline
\end{tabular}

Either chi-square test or Fisher's exact test was used. *time from abuse onset to the first interview, the suspect threatened the victim about consequences of disclosure in our sample involved elaborate statement analysis, we did not delve into the association between judgment of credibility and the content of testimony except for reporting consistency. Finally, only two variables, multiple abuse events and reporting consistency, were identified as effective predictors of higher credibility.

A Norwegian survey has shown that psychiatrists and police officers express a greater belief in children's capacities in reporting events such as abuse than other professionals. ${ }^{24}$ Two recent studies conducted in Israel reveal that trained child investigators (mostly social workers) judge allegations of CSA as credible in approximately $60 \%$ of cases, ${ }^{8,14}$ which is relatively lower than our credibility rate $(72.9 \%)$. The sample sources for both Israeli studies cited here were the national data files of cases referred for forensic interviews, as used here. In addition to the potential for individual and personal beliefs regarding child witnesses playing a role, we explain the results as follows. First, some studies have suggested that a girl's testimony is more likely to be considered credible., ${ }^{9} 12$ The disproportion in gender composition ( $89.6 \%$ females) in our sample could have been responsible for increasing the number of perceived credible cases. Second, a prior study has shown that an evidence-based investigative interviewing protocol can facilitate the assessment of credibility. ${ }^{4}$ Most of the interviewers (district prosecutor) in our program are required to follow such a protocol and try their best to avoid the use of leading questions during interviews. Third, credibility assessments in the abovementioned Israeli studies were mainly based on children's statements in investigative interviews. Here, MDT of the psychiatry department might receive information about an abusive event from the victim, family members, or school teachers, as appropriate. These collateral materials might contribute to an increase in the probability of abuse allegations considered credible. Finally, a pre-investigation disclosure is highly predictive of a disclosure during a forensic interview. ${ }^{25}$ In our sample, $86 \%$ of the victims gave an initial disclosure before their cases were reported to authorities, which may reflect the child's willingness to disclose the details of the event during the formal interview. ${ }^{26}$

Our study failed to demonstrate that children of younger age ( $<7$ years) and with intellectual disability (IQ $<70)$ can reduce the likelihood of a credible judgment made by the child psychiatrist. This contradicts previously established findings on this subject. ${ }^{8,9,12,14}$ The forensic interviewers in our study followed the structured National Institute of Child Health and Human Development (NICHD) ${ }^{19,27}$ protocol as closely as possible, which recommends the use of free-recall prompts. Moreover, prior research has indicated that children as young as 4 years old can provide plenty of forensically critical information about abusive events in response to free-recall prompts. ${ }^{28}$ 
Table 2. Logistic regression analysis predicting credibility $(\mathrm{N}=95)$

\begin{tabular}{lrrrrr}
\hline \multicolumn{1}{c}{ Predictors } & \multicolumn{1}{c}{$\mathrm{B}$} & \multicolumn{1}{c}{ SE } & OR & 95\% CI & p \\
\hline Age at forensic interview (<7 years vs. $\geq$ 7 years) & -0.36 & 0.76 & 0.70 & $0.16-3.07$ & 0.635 \\
Caregiver (both parents vs. others) & 0.90 & 0.74 & 2.46 & $0.58-10.49$ & 0.223 \\
Familiarity with the suspect (yes vs. no) & -0.37 & 0.85 & 0.69 & $0.13-3.60$ & 0.658 \\
Number of abuse events (multiple vs. single) & 1.80 & 0.90 & 6.05 & $1.04-35.34$ & 0.046 \\
Suspect's threat (yes vs. no) & 19.03 & 11180.54 & $1.837 \mathrm{E} 8$ & - & 0.999 \\
Report consistency (yes vs. no) & 3.71 & 0.87 & 40.82 & $7.46-223.50$ & $<0.001$ \\
\hline
\end{tabular}

B: regression coefficient, CI: confidence interval, OR: odds ratio, SE: standard error of the coefficient

Based on this, when we lowered the cut-off values for age ( $\leq 4$ years vs. $>4$ years) or IQ ( $\leq 50$ vs. $>50$, which is the lower limit of mild mental retardation as defined by DSM-IV-TR), ${ }^{20}$ the associations between the two variables and credible judgment became statistically significant in the binary analyses $(\mathrm{p}=0.004$ and 0.03 , respectively). These changes may suggest that the use of such a structured interview protocol can further benefit the psychiatrist's credibility assessment regarding children aged $>4$ years or those having a mild intellectual disability (defined as $50<\mathrm{IQ}<70$ ).

The results from the logistic regression model showed that multiple abuse events could predict a significantly credible judgment. Early attempts at exploiting this factor have shown inconsistent findings. ${ }^{8,14,29}$ An experimental study demonstrated that young children who reported the details of a repeated event were judged as less credible than those who reported details of a one-time event. ${ }^{30}$ The authors of that study argued that "repeat-event children" were deemed less consistent and confident. Likewise, one Korean survey that assessed children aged 8-13 years who were sexually abused demonstrated that single-event abuse allegations are more credible than multiple-event abuse allegations. ${ }^{31}$ In that research, the suspects of a single event were mostly strangers, which could reduce the impact of family dynamics with regard to motivation for disclosure. Compared with the Korean study, the mean age of our children is younger ( $7.03 \pm 3.53$ years). In our study, $62.5 \%$ of perpetrators of a single abuse event were also strangers. We infer that it is more difficult for young children to describe the details of a one-time abuse event, especially one caused by a stranger. Hence, for assessing credibility in the reports by young children, cognitive competence might be relatively more important than other psychosocial factors.

Children's report consistency was a significant predictor $(\mathrm{OR}=40.82)$ for higher credibility in the present study. Conversely, inconsistencies are commonly viewed as a reflection of low accuracy or falsehood. ${ }^{32}$ From the perspective of child psychiatrists, we can assume that consistency is equal to accuracy. It can be particularly common to detect inconsistencies among children's reports when they repeatedly report a per- sonal experience. ${ }^{33,34}$ This phenomenon may emerge for a variety of reasons. First, children may remember some new information when repeatedly asked the same question. ${ }^{33}$ In our study, children with consistent reports were older than those with inconsistent reports ( 7.66 years vs. 6.03 years, $\mathrm{p}<0.05$ ). This suggests that younger children tend to be more inconsistent, and this warrants further discussion in written forensic psychiatric reports.

With regards to the characteristics of the familial context, we did not find that the caregiver was a significant predictor for perceived credibility in the final regression model. Nevertheless, in the bivariate analyses, care by both parents at the time of interview was found to significantly decrease the probability of a credible judgment. The finding partly contradicts some past studies, ${ }^{8,10,35}$ where it has been found that the possible presence of a divorce or custody dispute is associated with the subsequent judgment not to substantiate the allegations. However, due to the low sample size in the current study, the interpretation of this outcome may be limited.

The present study has several other limitations which should be noted. First, we recorded credibility entirely based on professional judgments made in written forensic psychiatric reports. There are limitations in the reliability of information acquired from children as well as their parents and school teachers. Second, a discussion regarding the impact of the interviewer's dimensions (such as interviewing skills) on the credibility assessment was not included within the scope of this study. Third, all our children were referred by the authorities. Thus, the generalization of the results to other populations under different settings may be restricted. Finally, the number of cases is limited.

In conclusion, the rate of CSA allegations judged as credible in this study is slightly higher than those reported in prior studies. From the perspective of child psychiatry in Taiwan, the methods of credibility judgment for CSA allegations may differ from those adopted in previous studies. In Taiwan, child psychiatrists often pay more attention to children's language competencies than possible psychosocial factors while assessing the credibility of witnesses. One likely reason for this 
finding is that age of the current study sample was relatively younger than that used in other studies. However, we cannot exclude the impact of cultural differences (e.g., a reduced display of emotions by children during a forensic interview) on credibility assessment. Further research is needed on how mental health professionals use a structured methodology to explore the factors influencing the credibility of children and their testimony and whether cultural differences exist in the context of credibility assessment.

\section{Acknowledgments}

The authors thank all colleagues who contributed to the program in the present study (Early Forensic Psychiatric Evaluation). We are also grateful to Dr. Chung Chang for assistance with statistical analysis.

\section{REFERENCES}

1. Bays J, Chadwick D. Medical diagnosis of the sexually abused child. Child Abuse Negl 1993;17:91-110.

2. Lamb ME. Child witnesses: recent research on children's accounts of forensically relevant experiences. Appl Dev Sci 1999;3:2-5.

3. Lipian MS, Mills MJ, Brantman A. Assessing the verity of children's allegations of abuse: a psychiatric overview. Int J Law Psychiatry 2004; 27:249-263.

4. Hershkowitz I, Fisher S, Lamb ME, Horowitz D. Improving credibility assessment in child sexual abuse allegations: the role of the NICHD investigative interview protocol. Child Abuse Negl 2007;31:99-110.

5. Ceci SJ, Crotteau-Huffman ML. How suggestible are preschool children? Cognitive and social factors. J Am Acad Child Adolesc Psychiatry 1997;36:948-958.

6. Chahal K, Cassidy T. Deception and its detection in children: a study of adult accuracy. Psychol. Crime Law 1995;1:237-245.

7. Vrij A. Criteria-based content analysis: a qualitative review of the first 37 studies. Psychol Public Policy Law 2005;11:3-41.

8. Melkman EP, Hershkowitz I, Zur R. Credibility assessment in child sexual abuse investigations: a descriptive analysis. Child Abuse Negl 2017;67:76-85.

9. Cross TP, Finkelhor D, Ormrod R. Police involvement in child protective services investigations: literature review and secondary data analysis. Child Maltreat 2005;10:224-244.

10. Haskett ME, Wayland K, Hutcheson JS, Tavana T. Substantiation of sexual abuse allegations: factors involved in the decision-making process. J Child Sex Abus 1995;4:19-47.

11. London K, Bruck M, Wright DB, Ceci SJ. Review of the contemporary literature on how children report sexual abuse to others: findings, methodological issues, and implications for forensic interviewers. Memory 2008;16:29-47.

12. Wood B, Orsak C, Murphy M, Cross HJ. Semistructured child sexual abuse interviews: interview and child characteristics related to credibility of disclosure. Child Abuse Negl 1996;20:81-92.

13. Voogt A, Klettke B. The effect of gender on perceptions of credibility in child sexual assault cases: a systematic review. J Child Sex Abus 2017; 26:195-212.

14. Hershkowitz I, Melkman EP, Zur R. When is a child's forensic statement deemed credible? A comparison of physical and sexual abuse cases. Child Maltreat 2018;23:196-206.

15. Davies M, Rogers P. Perceptions of blame and credibility toward victims of childhood sexual abuse: differences across victim age, victimperpetrator relationship, and respondent gender in a depicted case. J
Child Sex Abus 2009;18:78-92.

16. Reynolds LL, Birkimer JC. Perceptions of child sexual abuse: victim and perpetrator characteristics, treatment efficacy, and lay vs. legal opinions of abuse. J Child Sex Abus 2002;11:53-74.

17. Bernet W. Practice parameters for the forensic evaluation of children and adolescents who may have been physically or sexually abused. J Am Acad Child Adolesc Psychiatry 1997;36(10 Suppl):37S-56S.

18. Wang LH, Lu T, Tsai CH. Children's disclosure of sexual abuse during early forensic psychiatric evaluation in Southern Taiwan. J Formos Med Assoc 2016;115:1069-1075.

19. NICHD protocol \& translated versions. Available at: http://nichdprotocol.com/the-nichd-protocol/. Accessed April 29, 2018.

20. American Psychiatric Association. Diagnostic and Statistical Manual of Mental Disorders- Fourth Edition, Text Revision. Washington, DC: American Psychiatric Press; 2000.

21. Wechsler D. The Wechsler Intelligence Scale for Children- Fourth Edition. San Antonio, TX: NCS Pearson; 2003.

22. Wechsler D. Wechsler Preschool and Primary Scale of Intelligence-Revised. San Antonio, TX: Psychological Corporation; 1989.

23. Corwin D. Early Diagnosis of Child Sexual Abuse: Diminishing the Lasting Effects. In: Wyatt G, Powell G, Editors. The Lasting Effects of Child Sexual Abuse. Newbury Park, CA: SAGE, 1988, p. 251-269.

24. Melinder A, Goodman GS, Eilertsen DK, Magnussen S. Beliefs about child witnesses: a survey of professionals. Psychol Crime Law 2004;10: 347-365.

25. Keary K, Fitzpatrick C. Children's disclosure of sexual abuse during formal investigation. Child Abuse Negl 1994;18:543-548.

26. London K, Bruck M, Ceci SJ, Shuman DW. Disclosure of Child Sexual Abuse: a Review of the Contemporary Empirical Literature. In: Pipe M, Lamb ME, Orbach Y, Cederborg A, Editors. Child Sexual Abuse: Disclosure, Delay and Denial. Mahwah, NJ: Lawrence Erlbaum, 2007, p.11-39.

27. Lamb ME, Orbach Y, Hershkowitz I, Esplin PW, Horowitz D. A structured forensic interview protocol improves the quality and informativeness of investigative interviews with children: a review of research using the NICHD investigative interview protocol. Child Abuse Negl 2007;31:1201-1231.

28. Lamb ME, Sternberg KJ, Orbach Y, Esplin PW, Stewart H, Mitchell S. Age differences in young children's responses to open-ended invitations in the course of forensic interviews. J Consult Clin Psychol 2003; 71:926-934.

29. Thoennes N, Tjaden PG. The extent, nature, and validity of sexual abuse allegations in custody/visitation disputes. Child Abuse Negl 1990;14: 151-163.

30. Connolly DA, Price HL, Lavoie JA, Gordon HM. Perceptions and predictors of children's credibility of a unique event and an instance of a repeated event. Law Hum Behav 2008;32:92-112.

31. Kim TK, Choi S, Shin YJ. Psychosocial factors influencing competency of children's statements on sexual trauma. Child Abuse Negl 2011;35: 173-179.

32. Leippe MR, Manion AP, Romanczyk A. Eyewitness persuasion: how and how well do fact finders judge the accuracy of adults' and children's memory reports? J Pers Soc Psychol 1992;63:181-197.

33. Peterson C, Moores L, White G. Recounting the same events again and again: children's consistency across multiple interviews. Appl Cogn Psychol 2001;15:353-371.

34. Hamond NR, Fivush R. Memories of Mickey Mouse: young children recount their trip to Disneyworld. Cogn Dev 1991;6:433-448.

35. Bowen K, Aldous MB. Medical evaluation of sexual abuse in children without disclosed or witnessed abuse. Arch Pediatr Adolesc Med 1999; 153:1160-1164. 